\title{
Novel antimicrobial fights TB resistance
}

Otsuka Pharmaceutical's delamanid (OPC-67683), a nitrodihydroimidazooxazole derivative, has demonstrated activity in patients with multidrug-resistant tuberculosis (MDR-TB) in a Phase II trial (N. Engl. J. Med. 366, 2151-2160; 2012).

TB is a potentially fatal infectious lung disease, most commonly caused by Mycobacterium tuberculosis. Effective treatment of TB is complicated, typically involving a cocktail of antimicrobials administered for many months, leading to non-compliance and selection of resistance. In addition, initial drug resistance testing is not performed for most patients. As a result, patients receive inappropriate treatment, which makes existing resistance worse, and this is now a key contributor to the increasing prevalence of MDR strains. Richard Chaisson, Professor of Medicine, Epidemiology and International Health, and Director, Centre for TB Research, Johns Hopkins University, Maryland, USA, explains further: "Creation of drug resistance is continuing around the world owing to weak TB treatment programmes as well as inadequate detection of drug resistance in newly diagnosed patients, which leads to improper management, amplification of resistance and transmission of infection to others." Alimuddin Zumla, Professor of Infectious Diseases and International Health, University College London Medical School, UK, adds: "With over 500,000 cases of MDR-TB worldwide, only $1 \%$ of patients with MDR-TB are estimated to be on appropriate drug treatment, highlighting the necessity for new TB drugs and better drug regimens."

There are several issues hampering the development of novel TB therapies and treatment approaches. "There is a need for identification of both novel targets to overcome resistance and drugs that can target persisting organisms, which currently make TB therapy so prolonged and serve as the source of relapse. In addition, it is clear that combination drug regimens are essential, and understanding which agents can be put in a regimen that will safely and effectively improve outcomes is a major obstacle that is not yet being adequately addressed," says Chaisson. However, as Zumla notes: "The few companies that have made the commitment to develop a new TB drug despite the very limited potential returns have been reluctant to have their drug studied for use in new combinations by not-for-profit sponsors outside of the narrow pathway they have taken for accelerated marketing approval. Multiple consortia must work together with funders, industry and governments to rapidly evaluate, license and introduce new drugs and drug regimens."

Of the handful of new or repurposed drugs currently in clinical trials for TB, delamanid, which inhibits the synthesis of mycolic acid (a major component of the mycobacterium cell wall), is one of only two novel agents to successfully complete Phase II efficacy trials, the other agent being bedaquiline (TMC-207, Janssen Pharmaceuticals), a diarylquinoline that targets ATP synthesis.

In the recently published trial, 481 MDR-TB patients received $100 \mathrm{mg}$ or $200 \mathrm{mg}$ of delamanid or placebo, twice daily for 2 months, in combination with a background drug regimen. The primary efficacy end point was the proportion of patients with sputum-culture conversion, defined as a series of five or more consecutive cultures that were negative for growth of M. tuberculosis in a liquid broth medium. Of the patients receiving the $100 \mathrm{mg}$ or $200 \mathrm{mg}$ delamanid doses, $45.4 \%$ and $41.9 \%$, respectively, had sputum-culture conversion in liquid broth at 2 months, compared with $29.6 \%$ of patients receiving placebo. Importantly, most adverse events were mild to moderate in severity and were evenly distributed between groups.

On the basis of these promising results, delamanid is now in a Phase III trial. "The fact that delamanid administered with the background drug regimen for MDR-TB enhanced and accelerated sputum-culture conversion is exciting and promising, bringing to light possibilities that the drug could reduce the lengthy duration of treatment, improve patient compliance and also reduce the duration for which the patient remains sputum-positive and infectious," says Zumla. "Delamanid has considerable potential, but needs to be studied in combination with other agents to determine which regimens will be safest and most effective," concludes Chaisson.

Sarah Crunkhorn 